\title{
Design of Audio Player Based on Spontaneous EEG
}

\author{
Jinghai Yin and Yuhua Chen \\ Institute of Information Technology, Jiangxi University of Technology, Nanchang 330098, China
}

Keywords: EEG; Audio player; BCl

\begin{abstract}
This article describes the design of a device which can according to the characteristics of brain waves in different users to choose the most suitable for the user to enjoy music, but also can automatically adjust the volume up to the user the most suitable to accept the extent of, all regulatory processes are by the system automatic implementation and completion of, making the playing process of brain waves to achieve adaptive.
\end{abstract}

\section{Introduction}

EEG based brain computer interface (BCI) systems can be used for people with disabilities to improve their quality of life. Applications of BCI systems comprise the restoration of movements, communication and environmental control. The most interesting thing in these applications is to control the roles in game by player's EEG. A volume of research has been performed on create information chain from brain to PC game by BCI system. Anton Nijholt(2008) Reported that Prototype BCI applications now appear in the domain of games and entertainment that aim at adapting and controlling a game using brain signals in addition to traditional physical and mental abilities.

In daily life, an audio playing device is we often want to use digital equipment, from a light, portable iPod to the computer's built-in playback software or is professional audio combination, the most basic control functions are basically the same, contain broadcast on the project selection and the regulation of the volume. For those who are not in action, or who want to listen to music, they rely on the changes in brain waves to regulate them.

The adaptive audio playback system mainly includes: the EEG signal acquisition device, a pre processing module, an electrode wire, a feature extraction module, feature recognition module, playback control interface, audio playback equipment, feature library and system control module. System through the EEG signal acquisition device collecting original EEG signals, and then through the pre processing module of signal processing, filter out some of the noise, and then extract its features after the feature library to do comparison for classification, and the identified results are converted into control signals to be transmitted to the audio playback device.

The system can determine whether the current user is suitable for appreciating which kind of music, whether it likes the current music, and then the automatic management of music tracks. Also for before going to bed to listen to music to help them improve the sleep of the user, the system will accord the real-time sleep EEG changes gradually to lower the volume until off playing system.

\section{Methods}

In this paper, we use short-term Fourier transform for the adaptive time-frequency analysis and energy entropy have been applied to feature extraction.

Shannon entropy. The conventional definition of the Shannon entropy was described in terms of the temporal distribution of signal energy in a given time window. The distribution of energy in a specified 
number of data values intervals was described in terms of the probabilities in signal space $\left\{p_{i}\right\}$ where $p_{i}$ was the probability that $X=a_{i}$, so entropy for discrete random variable $X$ was defined as

$$
H(X)=-\sum_{i} p_{i} \log \left(p_{i}\right)
$$

The entropy of a random variable reflected the degree of disorder that the variable possessed. The more uncertain the variable was, the greater its entropy.

Energy entropy. Short-term Fourier transform was a time-frequency analysis, which could analyze no stationary time-varying signals on frequency domain and time domain at the same time, so the dynamic change of signal energy with time could be observed. Energy Entropy defined on the basis of this could characterize signal complexity with the changes in time, and also many of the characteristics in frequency domain, which had a good time-frequency local capabilities.

Let $E_{1}, E_{2}, \wedge E_{m}$ represented energy distribution on the $m$ frequency bands. Then signal energy $E$

equaled to the sum of $E_{j}$ in a time window $E=\sum_{j=1}^{m} E_{j}$, where $E_{j}=\sum_{k}\left|D_{j}(k)\right|^{2}$, where $D_{j}$ was spectrum on the jth frequency band. Let

$$
\begin{aligned}
& p_{j}=E_{j} / E \\
& \text { then } \sum p_{j}=1 \text {, The definition of energy entropy was corresponding to: } \\
& W_{e}=-\sum_{j} p_{j} \log p_{j}
\end{aligned}
$$

\section{System workflow}

The workflow diagram of the system is as follows: 


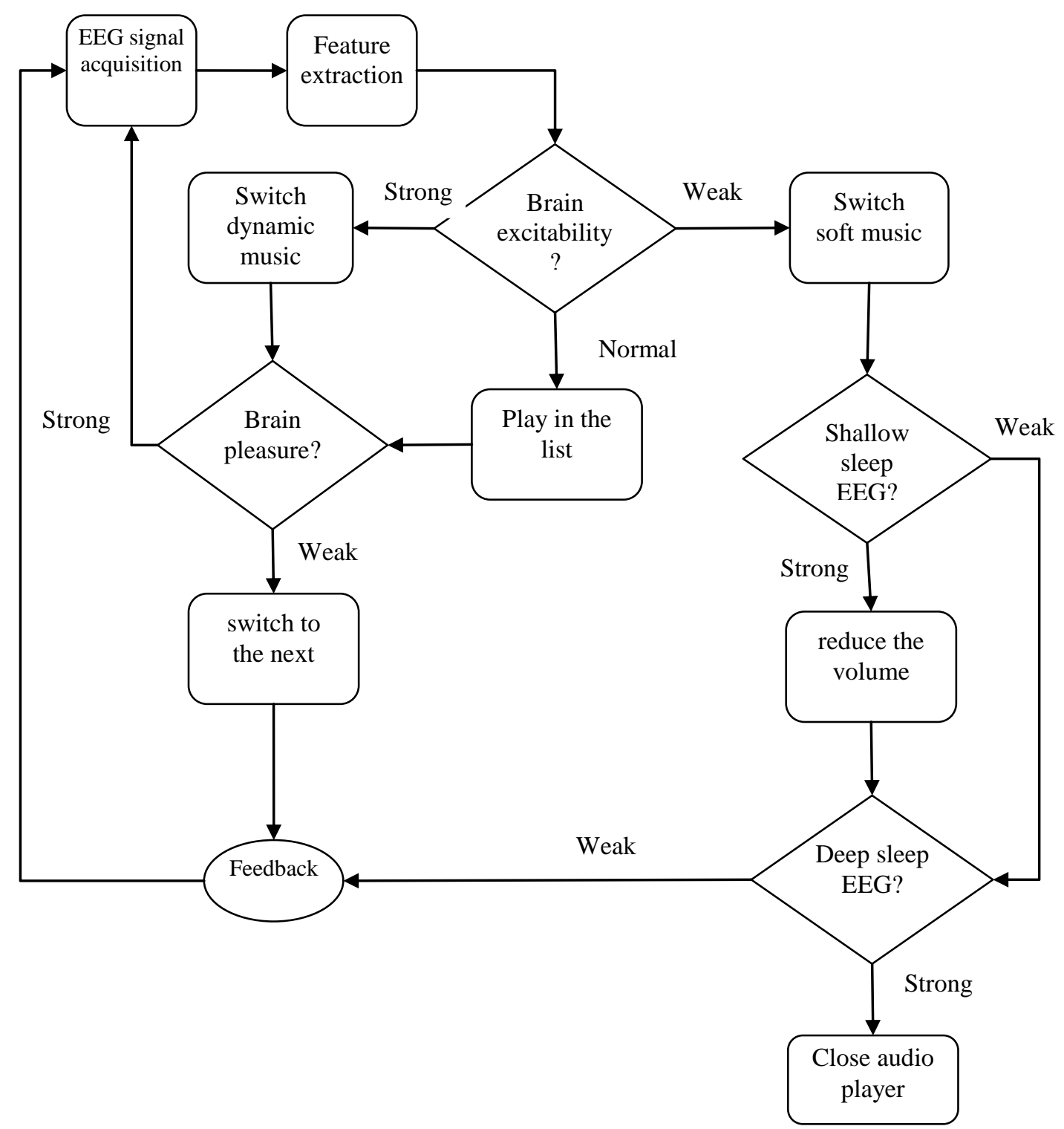

Fig. 1 workflow chart

As shown in Fig. 2, when we open the audio player system, and bring up the EEG acquisition device. System through the identification of brain waves to determine the current user's brain excitability to choose to play the right song, when the brain is excited at the high degree, auto play music more sporty, when brain excitability low, playing soft music, otherwise, in accordance with the normal playlist playback.

When the system determines the current user's brain excitability for high or medium, the system will play with respect to the music, then judge the brain's pleasure in. If the brain is enjoyable, proper selection of music, the system will continue to play, if the user's pleasure is decreased, then the system will interrupt playback then automatically switching a first appropriate music playback.

When the system determines the current user's brain excitability is low, the system will play soft music, at this time the user may in a state ready to sleep, system will determine the brain of shallow sleep EEG, if the waveform is obvious that users are likely to enter the shallow sleep, the system will 
gradually reduce the volume, and then judge whether deep sleep EEG obviously, if strengthen the user has entered a deep sleep, the system will automatically stop playback.

\section{Design of System architecture}

The external structure of the system is shown in the following figure:
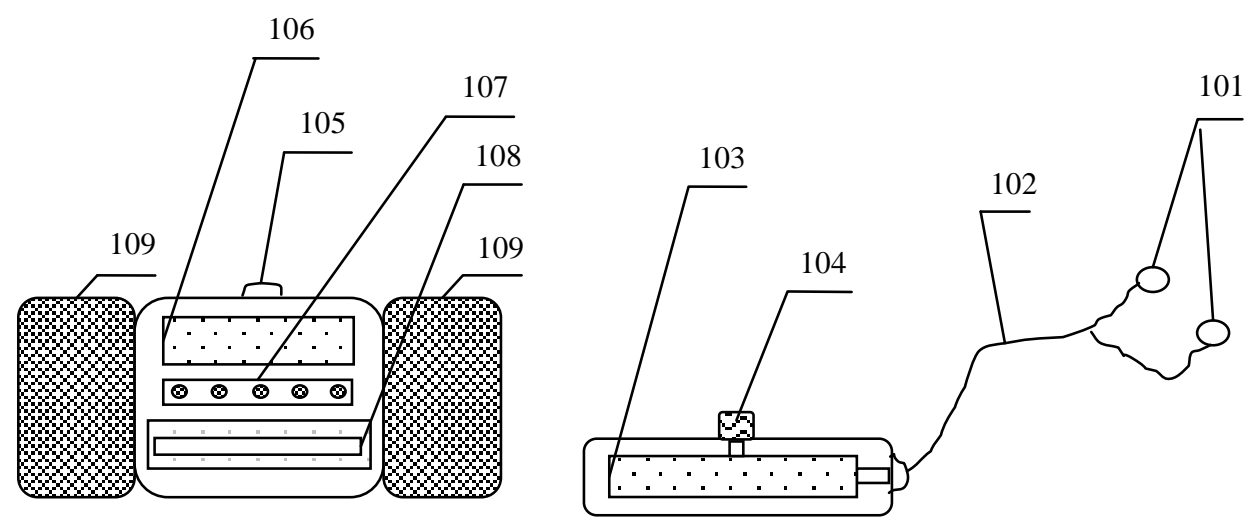

Fig. 2 External structure diagram

As shown in Fig. 2,

The adaptive audio player is composed of two parts, including the player part and the brain electrical signal acquisition and analysis. The two part is the communication between the player and the brain.

Brain electrical signal acquisition and analysis is made up of two dry electrodes 101, two signal wires 102, a signal analysis and recognition module 103 and a Bluetooth transmitter module 104. Dry electrode 101 is an alloy material, which is attached to the surface of the skin. Two electrodes placed on the user's left prefrontal cortex and right prefrontal area system through the two dry electrode to collect the original EEG signals and by two signal conductors 102 will the conduction to analysis identify module (103) of the signal analysis was performed to identify, the module integrated a / D conversion, signal pre processing, signal analysis, feature classification and recognition functions. By extracting information about the brain's excitement and attention in the brain electrical signal, the information is converted to one dimensional data through Bluetooth transmitter 104 to the audio player.

Player is composed of 105- Bluetooth receiver, 106- LCD screen, 107- player controller, 108-CD driver, 109- speaker. Bluetooth receiver 105 placed in the broadcast of the top, liquid crystal display 106 for displaying state and parameters of the player, playing controller 107 to control music playback, CD driver 108 for playing CD music, sound box 109 for the sound of the outside.

\section{Discussion}

The system can according to the characteristics of brain waves in different users to choose the most suitable for the user to enjoy music, but also can automatically adjust the volume up to the user the most suitable to accept the extent of, all regulatory processes are by the system automatic implementation and completion of, making the playing process of brain waves to achieve adaptive. 


\section{Acknowledgment}

This work was supported by Jiangxi province department of science and technology support project [20142bbe50030] and Natural Sciences Project of Jiangxi Science and Technology Department [20122BAB201049]. The authors are grateful for the anonymous reviewers who made constructive comments.

\section{References}

[1] G. Pfurtscheller, C. Guger, G. Müller, G. Krausz, and C. Neuper, Brain oscillations control hand orthosis in a tetraplegic," Neurosci. Lett., vol. 292, pp. 211-214, 2000..

[2] S.M. Stevens and S. Saldamarco (Eds.) BCI for Games: A 'State of the Art' Survey ICEC 2008, LNCS 5309, pp. 225-228, 2008.

[3] Ince N.F., Arica S., Tewfik A., "Classification of single trial motor imagery EEG recordings with subject adapted nondyadi arbitrary time-frequency tilings," J Neural Eng, Vol. 3, pp. 235-244, 2006.

[4] Jianfeng Hu,Dan Xiao,Zhendong Mu. Application of Energy Entropy in Motor Imagery EEG Classification. International Journal of Digital Content Technology and its Applications,2009,3(2): 83-90

[5] R. Nicole, "Title of paper with only first word capitalized," J. Name Stand. Abbrev., in press.

[6] McFarland D.J., Wolpaw J.R., "Sensorimotor rhythm-based brain-computer interface (BCI): model order selection for autoregressive spectral analysis," J Neural Eng, Vol. 5, pp. 155-162, 2008.

[7] Ramoser H., Müller-Gerking J., Pfurtscheller G., "Optimal spatial filtering of single trial EEG during imagined hand movement," IEEE Trans Rehabil Eng, Vol. 8, pp. 441-446, 2000.

[8] Müller-Gerking J., Pfurtscheller G., Flyvbjerg H., "Designing optimal spatial filters for single-trial EEG classification in a movement task," Clin Neurophysiol, Vol. 110, pp. 787-798, 1999.

[9] Hoffmann U., Vesin J.M., Ebrahimi T., "Spatial filters for the classification of event-related potentials," Proc 14th Eur Symp Artif Neural Networks, pp. 47-52, 2006.

[10]Liao X., Yao D.Z., Wu D. et al, "Combining Spatial Filters for the Classification of Single-Trial EEG in a Finger Movement Task," IEEE Trans Biomed Eng, Vol. 54, pp. 821-831, 2007. 